\title{
EDITORIAL
}

\section{Fetal Neuropathology and Abnormal Motor Assessment_Fetal Akinesia Deformation Sequence: KANET is of Additional Value for the In Utero Diagnosis}

Hermann U Honemeyer ${ }^{1}$, Asim Kurjak ${ }^{2}$

\begin{abstract}
The fetal activity has a fundamental role in forming the fetal gestalt. Fetal movements produce biomechanical stress, with distinct biochemical effects determining the morphogenesis of the musculoskeletal apparatus. As a result of normal maturation of the fetal brainstem, and shaped by general movements (GMs) and increasing variability of highly differentiated isolated movements, the typical human fetal gestalt evolves. KANET scoring system allows quantitative and qualitative determination and analysis of fetal movements and gestalt during the second and the third trimesters. The diagnostic efficiency of KANET in detecting abnormal fetal behavior and neuromorbidity is demonstrated with two cases of fetal akinesia deformation sequence (FADS).

Keywords: Fetal akinesia deformation sequence, Fetal behavior, Fetal gestalt, Fetal neurology, Kurjak's antenatal neurodevelopment test. Donald School Journal of Ultrasound in Obstetrics and Gynecology (2019): 10.5005/jp-journals-10009-1601
\end{abstract}

\section{INTRODUCTION}

The research of fetal behavior has been stimulated and advanced by $3 \mathrm{D}$ and 4D ultrasound technology. With ever-improving image quality and frame rates, stunning insights into the fetal world provide an abundance of scientific material and have become a fascination for parents. While many researchers recognized the potential of 3D and 4D ultrasound for studying fetal activity, it is the merit of Asim Kurjak and his group to have observed and categorized normal fetal movements in longitudinal studies during pregnancy and, thus, given us a concept of normal fetal behavior (Kurjak et al.). " "Kurjak's Antenatal Neurodevelopment Test" (KANET), as the result of these studies, represents the first standardized easy-to-learn/easy-to-use sonographic scoring system which allows to measure the degree of deviation from normal fetal behavior in utero. The relevance of this scoring system becomes more than obvious in the clinical scenario of FADS, where the examiner is "struck" by total immobility of a fetus which may otherwise exhibit only minor structural or postural abnormalities. Here, the hint at a fatal neuropathology of the fetus comes essentially from the abnormal result of the fetal motor assessment. In this case study, KANET was applied in two cases of polyhydramnios with absent fetal movements/abnormal gestalt, and led to the diagnosis of FADS. Furthermore, this case study intends to highlight the key role of abnormal or absent fetal movement in the etiology of fetal morphological deformation.

\section{Case Description}

\section{Case 1}

A second gravida para 1, 40 years old, with spontaneous vaginal delivery at the term of her first child, had conceived spontaneously. She was referred to us at $30+2$ weeks for fetal biometry and Doppler. She had felt the first "quickening" at 18 weeks, but fetal movements had stopped abruptly at 26 weeks. Fetal movements had started 3 weeks earlier than in her first pregnancy, a rather common experience in multiparity; however, she emphasized that they were more frequent and vigorous in this second pregnancy.
${ }^{1}$ Department of Obstetrics and Gynecology/Fetal Medicine, Alzahra Hospital and NMC Healthcare, Sharjah, UAE

${ }^{2}$ Department of Obstetrics and Gynecology, Medical School University of Zagreb, Zagreb, Croatia; University Sarajevo School of Science and Technology, Sarajevo, Bosnia and Herzegovina

Corresponding Author: Hermann U Honemeyer, Department of Obstetrics and Gynecology/Fetal Medicine, Alzahra Hospital and NMC Healthcare, Sharjah, UAE, e-mail: dr.ulrich.ho@hotmail.com

How to cite this article: Honemeyer HU, Kurjak A. Fetal Neuropathology and Abnormal Motor Assessment-Fetal Akinesia Deformation Sequence: KANET is of Additional Value for the In Utero Diagnosis. Donald School J Ultrasound Obstet Gynecol 2019;13(3):88-93.

Source of support: Nil

Conflict of interest: None

Her previous medical, obstetric, and family history was unremarkable. In this second pregnancy, early pregnancy scans, first trimester nuchal translucency screening, and a detailed second trimester fetal anatomy scan had all been normal. On noticing decreased fetal movements at 26 weeks, she went to a hospital emergency where she was reassured.

Our sonographic findings at gestational age $30+2$ weeks: normal fetal anatomy. Biometry equivalent to $28+1$ weeks. Abdominal circumference (AC) minus 2.4 SD suggestive of intrauterine growth restriction (IUGR). Except for a cerebellum diameter of minus 2.2 SD, neurosonography was normal. Normal middle cerebral artery (MCA), umbilical artery (Umb.A), and uterine artery (UA) Doppler waveforms and resistance indices. Mild polyhydramnios. Abnormal biophysical profile due to the absence of fetal movements and breathing.

\section{Fetal "Gestalt" and Motor Assessment with 3D/4D Ultrasound Using KANET}

The fetus was in a breech position with extended legs. The tongue protruded from a slightly gaping mouth. The fetal profile 
showed moderate retrognathia. Bilaterally clenched fists. No fetal movements. The KANET score was 3 points.

At $30+3$ weeks, an uncomplicated amniocentesis was performed. The KANET score on this occasion 4 points. Unchanged fetal "Gestalt".

Repeated nonstress tests (cardiotocography) were characterized by complete loss of accelerations and variability.

Fetal cytogenetics showed 46, female karyotype (XX) without numerical or structural anomalies. The amniotic fluid evaluation for acetylcholinesterase, alpha-fetoprotein, and fetal infections (parvovirus B19-PCR) was normal.

At 33 weeks, polyhydramnios had turned severe. The patient suffered from breathing difficulties, back-and shoulder pain, and was unable to sleep. At $34+1$ weeks, an amniocentesis with the removal of 3,500 $\mathrm{mL}$ was performed, followed by the onset of labor. Because of unchanged breech position, she consecutively underwent LSCS at $34+2$ weeks. The newborn did not cry, did not breathe, was intubated on the third attempt 17' postpartum, and required controlled ventilation.

\section{Postnatal Findings}

Magnetic resonance imaging (MRI): global volume loss of the hemispheres and the cerebellum. Electroencephalography (EEG): diffuse low voltage and slow activity consistent with severe encephalopathy. US: bilateral intraventricular hemorrhage and increased echogenicity of bilateral basal ganglia.

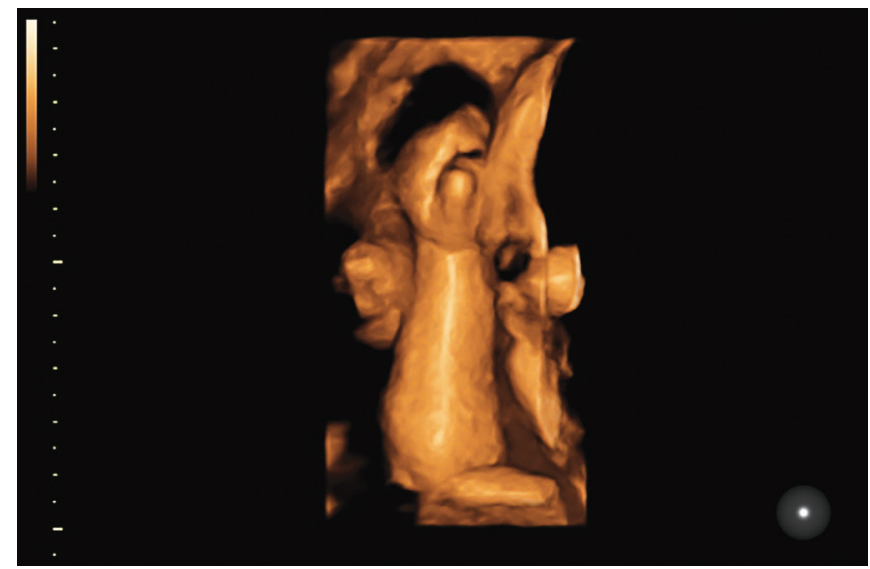

Fig. 1: Adducted thumb, also called the "neurological thumb", seen in various cases of severe neuropathology

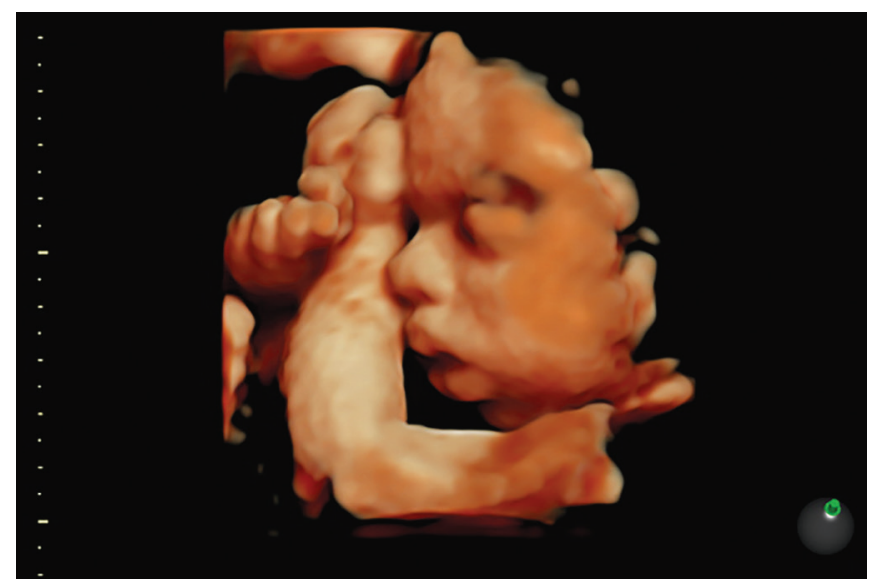

Fig 3: Profile view
The parents were counseled that the phenotype of the newborn with akinesia and severe contractures was suggestive of the Pena-Shokeir syndrome and that the postnatal findings meant severe neuromorbidity with poor prognosis. On day 5 , they wished compassionate withdrawal of ventilation, and the baby died $30 \mathrm{~min}$ after extubation.

Figures 1 to 4 show the fetus of case 1 at $30+2$ weeks, and in Figure 5 at $31+4$ weeks. Figure 6 shows the newborn in case 1 .

\section{Case 2}

Figures 7 to 9 show the fetus of case 2 at $30+0$ weeks. Figures 10 and 11 show the newborn in case 2 .

A 26-year-old primigravida was referred because of polyhydramnios at $30+1$ weeks gestational age (LMP). Medical history unremarkable, blood pressure, and BMI normal. Consanguineous marriage (1st-degree cousins).

\section{Sonographic Findings}

Moderate bilateral ventriculomegaly of $13.3 \mathrm{~mm}$, cisterna magna normal. Reduced gyration and flat Sylvian fissure. An increase in the nuchal fold thickness of $8.8 \mathrm{~mm}$, and the prenasal thickness of $6.2 \mathrm{~mm}$, generalized skin edema. Minimal filling of fetal stomach. Otherwise normal fetal anatomy. Biometry with estimated fetal weight on the 12th centile, $A C$ on the 3rd centile, and head circumference $(\mathrm{HC}) / A C$ ratio above the 99 th centile, indicating mild IUGR. Normal Doppler waveforms and resistance indices of $\mathrm{MCl}$ and $\mathrm{UA}$, with a cerebro-

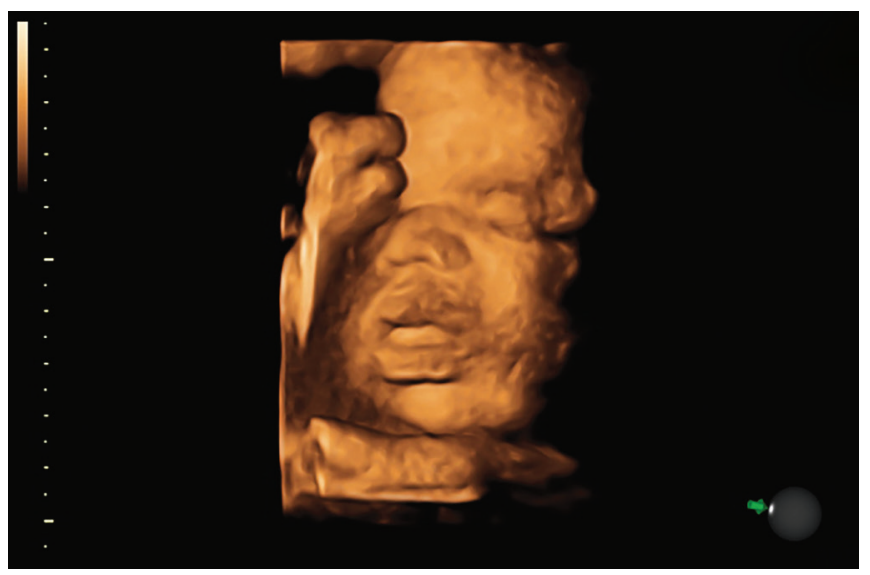

Fig. 2: Gaping mouth and slightly protruding tongue

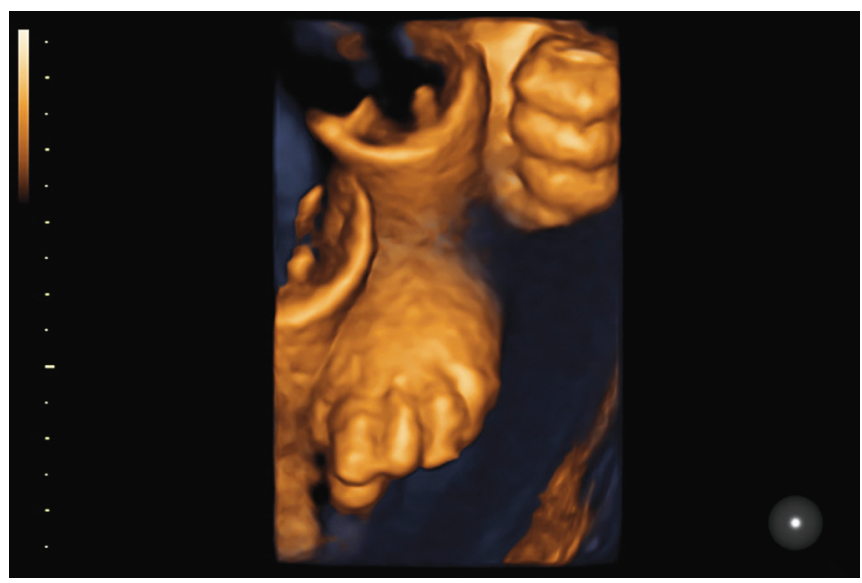

Fig. 4: Clenched fist and adducted big toe 


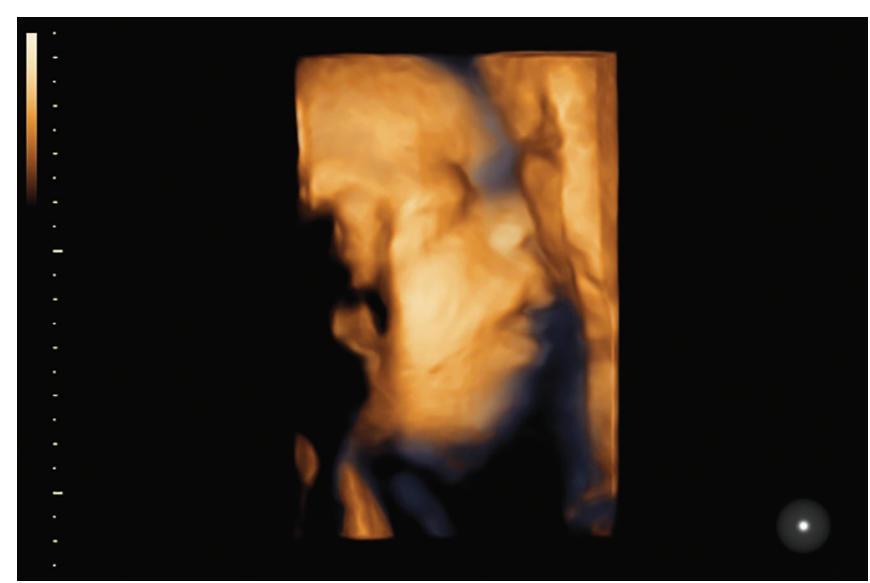

Fig. 5: Profile 9 days later at $31+4$ weeks

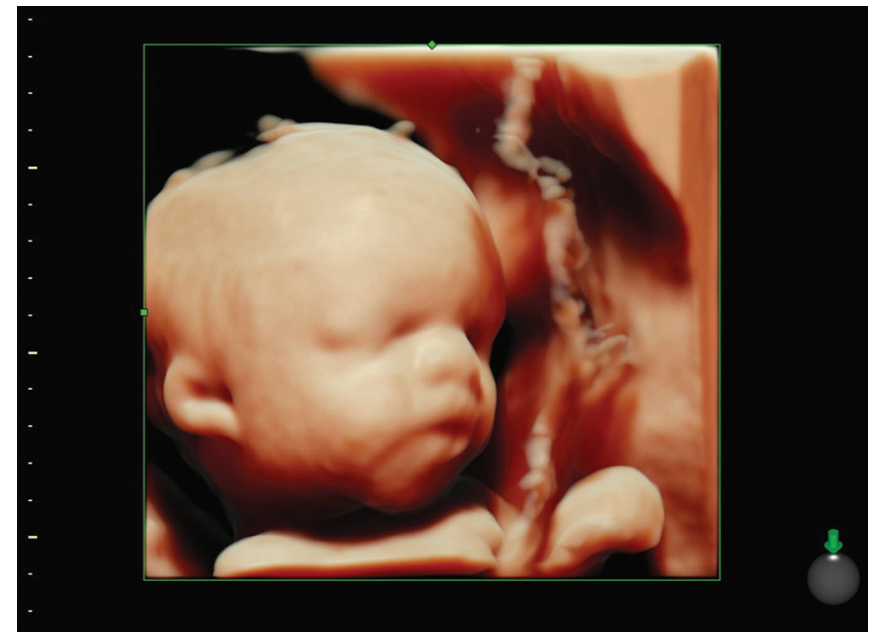

Fig. 7: Note the frozen facial expression, prenasal edema, and abnormal ears

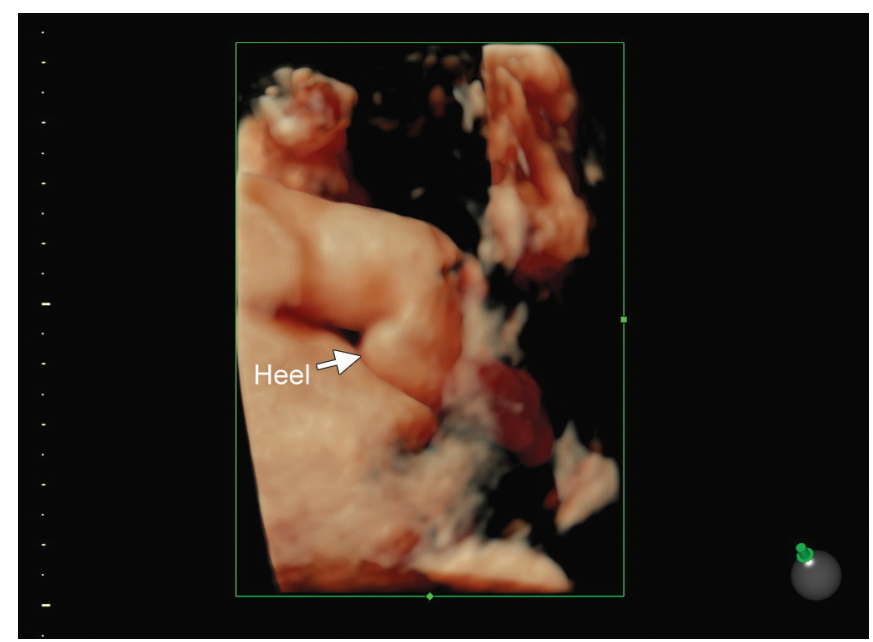

Fig. 9: Clubfoot

placental ratio (CPR) of 1.4. Abnormal biophysical profile due to fetal immobility and absent fetal breathing. Severe polyhydramnios.

\section{Fetal "Gestalt" and Motor Assessment with 3D/4D Ultrasound Using KANET}

Fetus in the breech position, adducted legs, and bent knees. Persisting flexion of both elbows, hyperflexion of the wrists, both

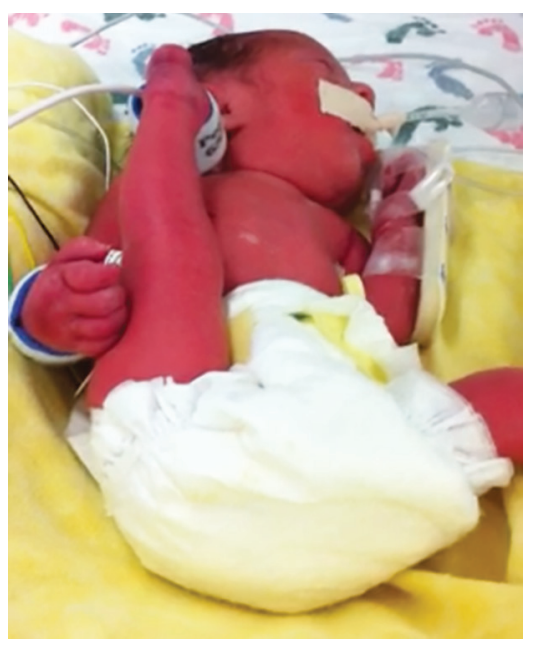

Fig. 6: Postpartum. Legs up and extended in fixation of intrauterine breech position

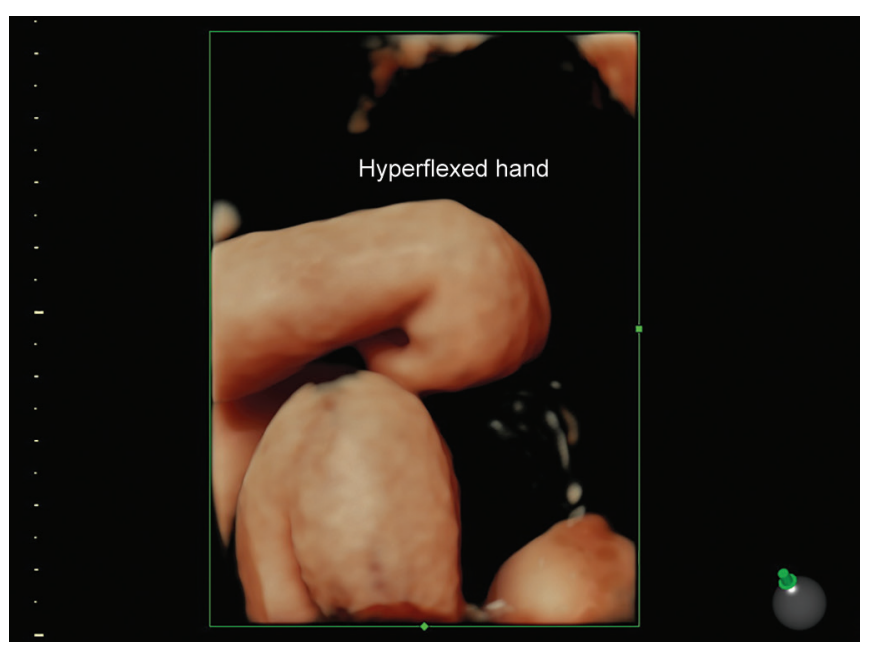

Fig. 8: Hyperflexed hand

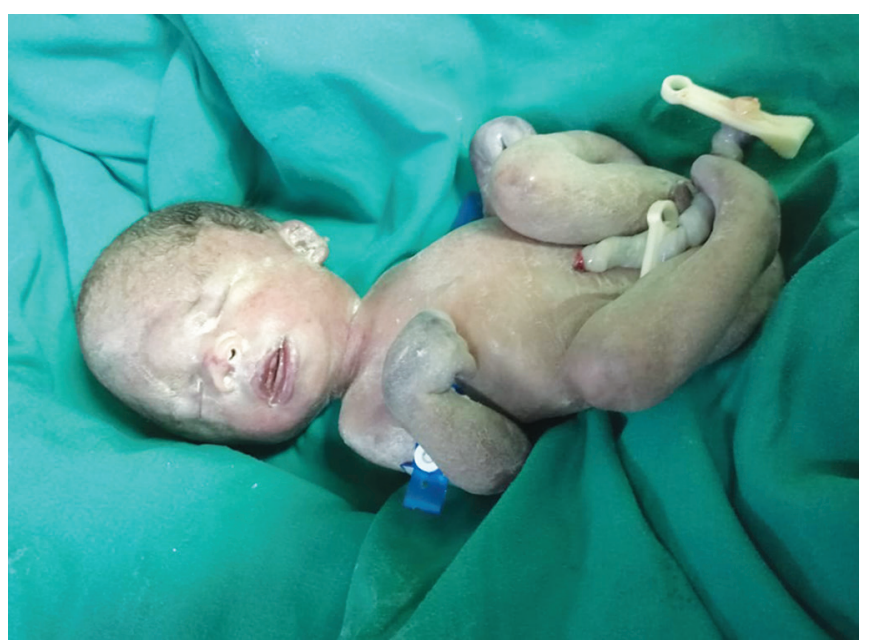

Fig. 10: Prenasal edema and hyperflexed hand

feet in the permanent club foot position. No fetal movements, unchanged fetal facies during the entire examination. KANET score abnormal with 2 out of 16 (normal HC and cranial sutures).

The nonstress test (cardiotocography) showed normal beat-tobeat variability and accelerations, but in an undulating baseline. 


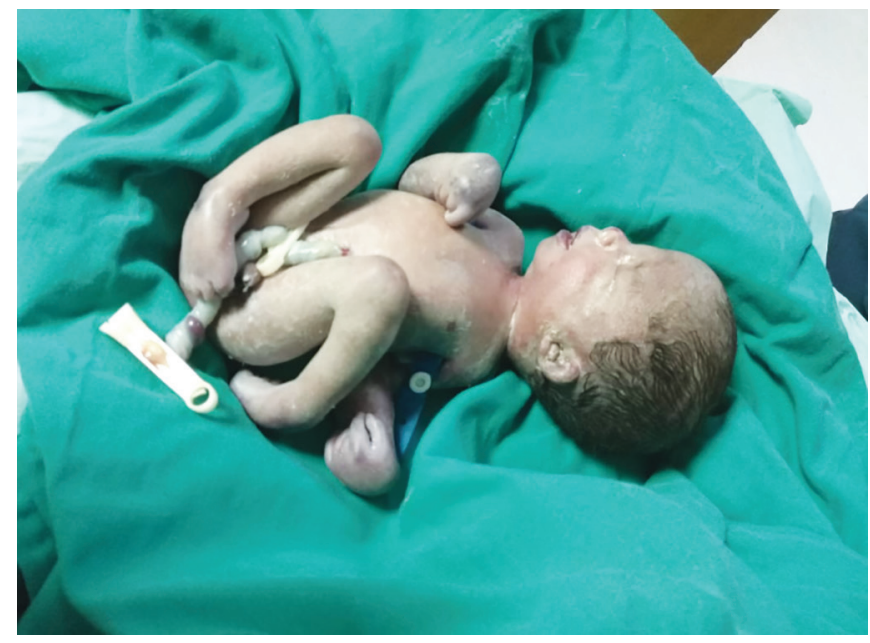

Fig. 11: Various deformations of the fetal gestalt

Findings were suggestive of FADS, and the parents were counseled accordingly. They decided to travel to their home country for delivery. There, the patient went into premature labor at 33 weeks due to severe polyhydramnios and-with the fetus still in the breach position-was delivered by LSCS. Neonatal resuscitation because of fetal apnoea was not successful, and the baby passed away shortly after delivery. The parents did not consent to a postmortem.

\section{Methodology of Antenatal Neuro- DeVelopment Test KANET}

Abnormal fetal neurobehavior can be scored using the KANET. Including the existing knowledge of structural criteria indicating neurological dysfunction such as reduced $\mathrm{HC}$ and overlapping cranial sutures (microcephaly), KANET uses a system of 8 fetal structural and behavioral parameters to evaluate the status of fetal neurodevelopment. The score for abnormal fetuses is from 0 to 5 , the borderline score is from 6 to 9 , and the normal score is 10 and above. The first part of the assessment in our two cases consisted of two-dimensional ultrasound examination (2D US) for the evaluation of the fetal position, growth, and anatomy, followed by Doppler ultrasound of the fetal circulation. Elevated Umb. A Doppler pulsatility index (UA PI) $>2$ standard deviations above mean for GA and reduced MCA pulsatility (MCA PI) index $<2$ standard deviations below the mean for GA, obtained in the absence of the fetal movements, were considered abnormal. The UA PI was measured in a free-floating loop of the umbilical cord. Measurements of the MCA PI were performed with color Doppler visualization of the circle of Willis. Doppler studies were followed by the assessment of fetal behavior applying the KANET test by means of four-dimensional ultrasound (4D US). In both cases, the examinations were performed by the same experienced operator with a GE Voluson E8 with RAB 4 3D/4D probe in case 1, and with a Voluson E10 with 3D/4D probe in case 2. The duration of the examinations was approximately $30-45$ minutes. All parameters of the KANET scoring system were evaluated quantitatively and qualitatively, assigning to each parameter scores from 0 to 2 . Scores from all parameters were summarized forming the total KANET score.

The inclined reader will find the KANET scoring system depicted and explained in detail in several other contributions to this issue.

\section{Discussion}

\section{Fetal Behavior and Gestalt}

Disturbances of the normal development of the fetal central nervous system are a root cause of alterations in the pattern of GMs and isolated movement. This has been demonstrated for an anencephalic fetus (Adenotopo et al.) ${ }^{2}$ and a fetus with lissencephaly (Yigiter et al.). ${ }^{3}$

We know today that GMs and isolated movements are important for the development of sensorimotor circuits and sensorimotor mapping in the fetus. But in a more basic sense, GMs-as all other spontaneous movements-are a precondition for the morphing of muscles, tendons, ligaments, cartilages, spindles, and bones into a normal human shape (Müeller), ${ }^{4}$ or "Gestalt" as we will call it here.

Going to a gym for "body shaping" is a reality of our adult life, and indeed, the principles which we use to shape our adult gestalt apply even more to the growing fetus.

Recently, an innovative study using the advanced technology of fetal cine-MRI could demonstrate that normal prenatal musculoskeletal development requires mechanical forces generated by active fetal movements (Verbruggen et al.). ${ }^{5}$ To quantify the stress and the strain in the fetal skeleton due to kicking during pregnancy, the following pipeline of computational techniques was applied to age-varying datasets during this study: (i) tracking the fetal movements of the joints from cine-MRI scans, (ii) finite element (FE) modeling of these movements to determine the resulting reaction force from the uterine wall, (iii) combining the above outputs in a musculoskeletal model which predicts the intramuscular forces required to generate the observed displacements, and (iv) application of these forces in an FE model of fetal limb geometries to calculate the resulting stress and strain. ${ }^{5}$ Cases were excluded where there was a known or suspected musculoskeletal abnormality, either on postmortem MRI or on subsequent autopsy. Six scans were included in this study, two from approximately 20 weeks gestation (a 19- and a 20-week), two from approximately 30 weeks gestation (two at 29 weeks), and two from approximately 35 weeks gestation (a 33-week and a 34-week). ${ }^{5}$ Based on their results, the authors postulate that "the stresses and strains on the fetal skeleton observed in this study act as biomechanical stimuli for limb development and morphogenesis". ${ }^{5}$

Similar postulates had been presented before by several other authors based on animal studies. ${ }^{6-8}$

Pathogenesis of abnormal fetal motor-activity, short "behavior", is better understood knowing that until delivery, subunits of the brainstem are the main regulators of all fetal behavioral patterns. The brainstem consists of the medulla oblongata, pons, and midbrain. These subunits form and mature in a caudal-to-rostral direction. Phylogenetically older structures, such as the medulla oblongata, will form and mature earlier in pregnancy than the pons and the midbrain. The medulla differentiates into many subnuclei and also gives rise to a variety of descending spinal motor tracts which-after myelinization-reflexively trigger limb and body movements. Five cranial nerves (VIII-XII) originate from the medulla and exert strong influences on gross body movements, heart rate, respiration, swallowing, and head turning. Hence, the pathophysiology of brainstem injuries ranges from the cessation of gross body movements, suspended fetal breathing, to fixed heart rate pattern, and impaired swallowing as a cause for polyhydramnios.

In a meta-analysis of 48 articles describing abnormal motility in all together 104 fetuses, De Vries et al., identified 14 types of 
hypo- and hyperkinetic movement disorders as a result of abnormal karyotype or congenital syndrome. ${ }^{9}$ The work-up of the differences in fetal phenotype expression and gestalt under conditions of hypo- and hyperkinesia is a topic of its own and deserves more scientific attention.

\section{FADS}

FADS is an umbrella term for neuromuscular-skeletal diseases of the fetus such as arthrogryposis multiplex congenita, lethal congenital contracture syndrome (LCCS), Pena-Shokeir syndrome type I (PSS I), and fetal brain death syndrome (FBDS). FADS limits itself to the description of both the underlying absence of fetal motor activity and the phenotypical "outcome," without a definition of causes. Therefore, now a brief description of each of these four entities.

Arthrogryposis is defined as a condition of congenital contractures in one or more joints. The term is not used as a specific diagnosis, but as a description of clinical symptoms, which may be found in more than 300 syndromes, and many other entities with unspecific defects. Teratogenic agents have been discussed in causing arthrogryposis, such as hyperthermia, hypoxia, ischemia, acidosis, infection, muscle relaxantia, prostaglandins, penicillamine, ergotamine, and enzyme inhibitors. Prenatal invasive genetic testing like chorion villus sampling (CVS) and early amniocentesis have also been linked to arthrogryposis. ${ }^{10}$

The Pena-Shokeir syndrome (OMIM 208150) was first reported in 1974 by Pena and Shokeir as an uncommon disease characterized by congenital multiple arthrogryposis, typical facies often with retrognathia, intrauterine growth retardation, polyhydramnios, camptodactyly, pulmonary hypoplasia, short umbilical cord, and lethality since these manifestations are usually so severe, that death occurs at birth or shortly after. However, survival until 9 years, with normal intelligence, has been described. ${ }^{11}$ "Pena-Shokeir" is not a diagnosis or a specific syndrome but rather a description of a phenotype produced by fetal akinesia or decreased in utero movements. This phenotype is present in a very heterogeneous group with a variety of anomalies and etiologies. It has, therefore, been recommended to use the term "fetal akinesia/hypokinesia deformation sequence." The etiology for the early cases was attributed to neuromuscular disease, with deformations and dysmorphism as a consequence of weakness or paralysis of the related motor units. In some cases, an abnormality of spinal cord motor neurons has been postulated. Recent observations have shown PSS resulting from blockage of the neuromuscular junction, in women who express antibodies against the fetal acetylcholine receptor. The PSS phenotype may also be caused by CNS dysfunction after intrauterine brain insults or brain malformation. ${ }^{12}$

Neuronal loss and ferrugination in the opercula, basal ganglia, thalami, and brainstem may be linked to the developmental form of the Foix-Chavany-Marie syndrome, or faciopharyngoglossomasticatory diplegia, characterized by deficits in swallowing and facial movements, and is possibly caused by an intrauterine hypoxic-ischemic injury to the developing central nervous system (CNS). ${ }^{13}$

According to others, the absence of fetal movements, caused by fetal neuropathy, leads to ankylosis of multiple joints, to absence of fetal breathing, depending on the insult timing in cases associated with pulmonary hypoplasia, and to absence of swallowing causing polyhydramnios, as well as to craniofacial anomalies due to absent movements of facial muscles. ${ }^{14}$
Lethal congenital contracture syndrome (LCCS) and the PenaShokeir syndrome have been discussed to have an autosomal recessive gene in up to $50 \% .^{15-17}$

By means of genome scans, the LCCS locus could be assigned to a defined region of chromosome $9 q 34$, in five affected individuals. ${ }^{18}$

Other genes have been identified to be responsible for various forms of the Pena-Shokeir syndrome: DOC 7 located in 4q16.2 and RAPSN located in 11q11.

In one epidemiological Finnish study between 1987 and 2002, all cases with multiple contractures were collected including live-born infants, stillbirths, and terminated pregnancies. Of all 214 cases, 92 ended as intrauterine fetal demise (68 selective pregnancy terminations (TOP), 24 were stillbirths), and 58 died in infancy. In 141 cases, the diagnosis was lethal arthrogryposis, with a prevalence of 1 in 6,985 (1.43/10,000 births). Of these, 59 had spinal cord pathology at autopsy and, thus, were of neurogenic origin. Thirty-nine cases were diagnosed as LCCS, clinically characterized by total immobility of the fetus at all ultrasound examinations (12 weeks or later), multiple joint contractures in both upper and lower limbs, and fetal death before 32 weeks of pregnancy. ${ }^{19}$

Prenatal ultrasonographic diagnosis of the Pena-Shokeir I syndrome with postnatal confirmation (case I) was previously described by Piotrowski and others in 2010. Cardiotocographic abnormalities, however, as in our case with a total absence of beatto-beat variability in repeated cardiotocography (CTG) sessions, have not been mentioned. ${ }^{20}$

The FBDS is a rare cause of a fixed fetal heart rate pattern and fetal immobility. Not many cases have been previously reported in the literature, only a few of them were diagnosed prenatally. All newborns died soon after delivery. ${ }^{21,22}$

FBDS is characterized by CTG demonstrating a fixed fetal heart rate (FHR) pattern. A detailed Doppler and US examination of the fetus may show initially normal neurosonographic findings as in case 1; however, progressive development of cerebral changes with ventriculomegaly may ensue. The clinical picture of FBDS includes polyhydramnios, total absence of fetal movements during biophysical profile (BPP), and eventually the cessation of cerebral blood flow.

A recent 10-year cohort study (Tjon et al.) focused on the timelines of abnormal phenotype development in akinesiadeformation sequence: using a motor assessment adapted from Donker et al., ${ }^{23}$ they evaluated 64 fetuses who fulfilled the study inclusion criteria. On the basis of the first advanced ultrasound assessment (AUE), FADS was suspected in 13 of 66 , arthrogryposis multiplex congenita (AMC) in 12 of 66 , bilateral pes equinovares (BPEV) in 40 of 66 , and the Holt-Oram syndrome in 1 of 66 . On the basis of the first motor assessment, the suspected diagnosis changed in 19 of 66 , in 13 of 66 worsening to FADS, 6 of 66 ameliorations from FADS, and confirmed FADS in 7 of 13. The result was 20 FADS, 7 $A M C$, and 38 BPEV. The second AUE in 44 fetuses showed additional contractures in 2 of the 8 FADS, and 1 intrauterine fetal death (IUFD). The second motor assessment changed the diagnosis in 3 of 43, 1 worsening from BPEV into FADS, 2 ameliorations from FADS, and confirmed FADS in 7 by the deterioration of motility. The result was 9 FADS, 6 AMC, and 29 BPEV. $^{24}$

This approach, based on understanding the importance of timelines of fetal brain development and of the impact of neuromorbidity on fetal movement, with consequences even of altered morphology, is essentially identical with the concept of Serial KANET (Honemeyer et al.), formulated, and applied in a study of fetal neurobehavior in high-risk pregnancies. ${ }^{25}$ 


\section{SUMmARY}

The fetal activity has a fundamental role in shaping the fetal gestalt. Movement patterns are laid out in the developmental program of the fetus and are visible already at 8 gestational weeks. They undergo meaningful modifications during pregnancy, influenced by maturational changes of the fetal CNS. This process is advanced by increasing fetal proprioceptive sensations and external stimuli. Fetal movements produce biomechanical stress, with distinct biochemical effects determining the morphogenesis of the musculoskeletal apparatus. As a result, the typical human fetal gestalt with an increasing variability of highly differentiated isolated and even goal-oriented movements evolves. The KANET scoring system allows quantitative and qualitative analysis and evaluation of fetal movements and gestalt during the second and the third trimester. In FADS, the KANET is diagnostic. At the same time, 4D ultrasound of the affected fetus helps parents to sensually grasp the seriousness of the fetal illness.

\section{References}

1. Kurjak A, Adenotopo W, et al. Normal standards for fetal Neurobehavioral developments-longitudinal quantification by four-dimensional sonography. J Perinat Med 2006;34(1):56-65. DOI: 10.1515/JPM.2006.007.

2. Adenotopo W, Kurjak A, et al. Behavior of an anencephalic fetus studied by $4 \mathrm{D}$ sonography. J Matern Fetal Neonatol Med 2005 Feb;17(2):165-168. DOI: 10.1080/jmf.17.2.165.168.

3. Yigiter AB, Goenenc G, et al. The assessment of fetal behavior of a fetus with lissencephaly by $4 \mathrm{D}$ ultrasound. Donald School JUltrasound Obstet Gynecol 2013;7(2):208-212. DOI: 10.5005/jp-journals-10009-1285.

4. Müller GB. Embryonic motility: environmental influences and evolutionary innovation. Evol Dev 2003;5:56-60. DOI: 10.1046/j.1525142X.2003.03009.x.

5. Verbruggen SW, Kaiz B, et al. Stresses and strains on the human fetal skeleton during development. J R Soc Interface 2018;15:20170593. DOI: 10.1098/rsif.2017.0593.

6. Brunt LH, Norton JL, et al. Finite element modelling predicts changes in joint shape and cell behaviour due to loss of muscle strain in jaw development. J Biomech 2015;48:3112-3122. DOI: 10.1016/ j. jbiomech.2015.07.017.

7. Nowlan NC, Murphy P, et al. A dynamic pattern of mechanical stimulation promotes ossification in avian embryonic long bones. J Biomech 2008;41:249-258. DOI: 10.1016/j.jbiomech.2007.09.031.

8. Nowlan NC, Dumas G, et al. Biophysical stimuli induced by passive movements compensate for lack of skeletal muscle during embryonic skeletogenesis. Biomech Model Mechanobiol 2012;11:207-219. DOI: 10.1007/ s10237-011-0304-4.

9. De Vries J, Fong BF. Changes in fetal motility as a result of congenital disorders: an overview. Ultrasound Obstet Gynecol 2007;29(5): 590-599. DOI: 10.1002/uog.3917.
10. Zimmer EZ, Jakobi $P$, et al. Cardiotocographic and sonographic findings in two cases of antenatally diagnosed intrauterine fetal brain death. Prenat Diagn 1992 Apr;12(4):271-276. DOI: 10.1002/ pd.1970120406.

11. Boesen PV, French CE. Acute respiratory distress in Pena-Shokeir syndrome. Ear Nose Throat J 2004 Nov;83(11):772-773. DOI: 10.1177/014556130408301115.

12. Kho N, Czarneck L, et al. Pena-Shokeir phenotype: case presentation and review. J Child Neurol 2002 May;17(5):397-399. DOI: $10.1177 / 088307380201700519$.

13. Hevner RF, Horoupian DS. Pena-Shokeir Syndrome phenotype associated with bilateral opercular polimicrogyria. Pediatr Neurol 1996 Nov;15(4):348-351. DOI: 10.1016/S0887-8994(96)00225-1.

14. Takada E, Koyama N, et al. Neuropathology of infant with PenaShokeir I syndrome. Pediatr Neurol 1994 May;10(3):241-243. DOI: 10.1016/0887-8994(94)90031-0.

15. Vuopala K, Herva R. Lethal congenital contracture syndrome: further delineation and genetic aspects. J Med Genet 1994 Jul;31(7):521-527. DOI: 10.1136/jmg.31.7.521.

16. Nousiainen HO, Kestila M, et al. Mutations in mRNA export mediator GLE1 result in fetal motoneuron disease. Nat Genet 2008 Feb;40(2):155-157. DOI: 10.1038/ng.2007.65.

17. Dzinovic A, Heljic S. Pena-Shokeir phenotype (fetal akinesia/ hypokinesia sequence. Med Arh 2006;60(6):383-385.

18. Maekela-Bengs $P$, Jaervinen $N$, et al. Assignment of the disease locus for lethal congenital contracture syndrome to a restricted region of chromosome $9 q 34$, by genome scan using five affected individuals. Am J Hum Genet 1998 Aug;63(2):506-516. DOI: 10.1086/301968.

19. Pakkasjaeri N, Ritvanen $A$, et al. Lethal congenital contracture syndrome(LCCS) and other lethal arthrogryposis in Finnland- an epidemiological study. Am J Med Genet A 2006 Sep 1;140A(17): 1834-1839. DOI: 10.1002/ajmg.a.31381.

20. Piotrowski K, Gizewska M, et al. Prenatal suggestion of Pena-Shokeir I syndrome postnatally confirmed. Arch Perin Med 2010;16(2):113-117.

21. Chen YT,HsuST, etal.Cardiotocographicand Dopplerultrasonographic findings in a fetus with brain death syndrome. J Obstet Gynecol 2006 Sep;45(3):279-282. DOI: 10.1016/S1028-4559(09)60243-6.

22. Iwasaki S, Morokuma S, et al. Acute onset antenatal fetal neurological injury suspected prenatally based on abnormalities in antenatal testing: a case report. J Matern Fetal Neonatal Med 2009 Dec;22(12):1207-1213. DOI: 10.3109/14767050903019619.

23. Donker ME, Eijckelhof BHW, et al. Serial postural and motor assessment of fetal akinesia deformation sequence (FADS). Early Hum Dev 2009;85(12):785-790. DOI: 10.1016/j.earlhumdev.2009.10.008.

24. Tjon JK, Tan-Sindhunata GM, et al. Fetal akinesia deformation sequence, arthrogryposis multiplex congenita, and bilateral clubfeet: Is motor assess- ment of additional value for in utero diagnosis? A 10-year cohort study. Prenat Diagn 2019;39:219-231. DOI: 10.1002/ pd.5411.

25. Honemeyer $\mathrm{U}$, Talic A, et al. The clinical value of KANET in studying fetal neurobehavior in normal and at-risk pregnancies.J Perinat Med 2012 Sep 28;1-11. 